\title{
A guide to implementing COVID-19 vaccine as a condition of employment in healthcare facilities
}

\author{
Joshua K. Schaffzin MD, $\mathrm{PhD}^{1}$ (1), A. Rekha K. Murthy $\mathrm{MD}^{2}$ (1), Valerie M. Deloney MBA ${ }^{3}$ (1), Trini Mathew MD, MPH ${ }^{4,5}$, \\ Erica Pettigrew MD, JD, MPH ${ }^{6,7}$, Ann Marie Pettis RN, BSN, $\mathrm{CIC}^{8}$, Kavita K. Trivedi MD ${ }^{9}$ and David J. Weber MD, MPH \\ ${ }^{1}$ Cincinnati Children's Hospital Medical Center, Cincinnati, Ohio, ${ }^{2}$ Cedars-Sinai, Los Angeles, California, ${ }^{3}$ Society for Healthcare Epidemiology of America, \\ Arlington, Virginia, ${ }^{4}$ HealthTAMCycle3, PLLC, Troy, Michigan, ${ }^{5}$ Beaumont Hospital, Royal Oak, Michigan, ${ }^{6}$ Orange County Health Department, Santa Ana, \\ California, ${ }^{7}$ University of North Carolina, Chapel Hill, North Carolina, ${ }^{8}$ University of Rochester Medicine, Rochester, New York and ${ }^{9}$ Trivedi Consults LLC, Berkeley, \\ California
}

To the Editor-Vaccination as a condition of employment (CoE) is an effective method for healthcare facilities to reduce the risk of disease transmission among patients, personnel, and their communities. Once a facility decides to enact such a policy, coordinated planning and logistics are needed to develop and implement it.

The Multisociety Statement on COVID-19 Vaccination as a Condition of Employment for Healthcare Personnel recommends COVID-19 vaccination as a CoE for healthcare personnel. ${ }^{1,2}$ This statement was led by the Society for Healthcare Epidemiology of America (SHEA), published in July 2021, and endorsed and referenced by many professional societies and leaders. ${ }^{3,4}$ As a subgroup of these authors, we have developed this companion statement and an online tool kit to assist facilities with planning and implementing a policy of COVID-19 vaccination for healthcare personnel (HCP).

\section{Inclusivity and fairness}

The decision regarding COVID-19 vaccine as a condition of employment $(\mathrm{CoE})$ requires consideration of a healthcare facility's goals, mission, and culture. Implementing a condition of employment requirement for COVID-19 vaccination requires employers to direct time and resources to engage HCP early and often to develop a fair, ethical, and transparent process based on the principles of diversity, equity, and inclusion. These factors are essential to progress toward the goal of a safer workforce through broad immunity while minimizing feelings of resentment or exclusion. Key elements necessary for organizational leadership during the strategic decision-making process include leadership transparency to garner trust and credibility among staff, an approach that includes and reflects the diversity of opinion and backgrounds of HCP, assessment of perceptions among HCP, and visible engagement and facilitation to seek input from stakeholders. While they are not likely to influence the requirement itself, facilities should acknowledge concerns at the organizational and individual levels for the smooth implementation of the policy, including COVID-19 vaccine safety and effectiveness, an institutional culture of respect and inclusivity, consistent and transparent exemption review, and collaboration with unions and other stakeholders. $^{1}$

Author for correspondence: Joshua K. Schaffzin, MD, PhD, E-mail: joshua.schaffzin@ cchmc.org

Cite this article: Schaffzin JK, et al. (2022). A guide to implementing COVID-19 vaccine as a condition of employment in healthcare facilities. Infection Control \& Hospital Epidemiology, 43: 1753-1754, https://doi.org/10.1017/ice.2021.405

\section{Access and support}

Operationalizing a CoE policy requires facilities to identify how to facilitate vaccination of HCP, tracking HCP vaccination status at facility and offsite locations, evaluating and approving medical and religious exemptions, and compensating HCP for vaccination and post-vaccination sick days without causing staffing shortages.

\section{Implementation tool kit}

SHEA has developed a tool kit for facilities implementing a policy of COVID-19 vaccination as a CoE. The kit is designed for facilities that have made the decision to require COVID-19 vaccination of employees and/or others functioning at their facilities, but it also can be utilized by those currently considering a requirement. As detailed in the Multisociety Statement, ${ }^{1}$ facilities can take actions to promote vaccination in the absence of a $\mathrm{CoE}$, to which the same implementation principles apply. Building on the SHEA/CDC Outbreak Response Training Program (ORTP) ${ }^{5}$, which provides guidance on how to mobilize an effective and successful outbreak response, ${ }^{5}$ this report is intended to provide a framework for healthcare facilities to plan and implement COVID-19 vaccination as a CoE for their HCP and as a source of readily available information, templates, and resources to help manage both strategic decision-making and establishing processes needed to operationalize a policy. Facilities with an existing performance improvement infrastructure will recognize many of the methodologies, and we have provided explanations and instructions for those less familiar. The tool kit is available publicly without registration (https://ortp. guidelinecentral.com/covid-19/).

Importantly, the inclusion of content and tools in this online resource does not indicate endorsement by SHEA, the authors, the authors' institutions, or other affiliated organizations. We welcome feedback, sharing, and suggestions; the tool kit is meant to be a "living" resource that may be updated as indicated, as long as the need for it remains.

COVID-19 vaccination as a CoE is an important tool for pandemic mitigation. We hope that SHEA members, partnering organizations' members, and the healthcare community will find the tool kit useful in their efforts to successfully implement a CoE policy.

\section{Acknowledgments.}

Financial support. No financial support was provided relevant to this article.

Conflicts of interest. All authors report no conflicts of interest relevant to this article. 


\section{References}

1. Weber DJ, Al-Tawfiq JA, Babcock HM, et al. Multisociety statement on COVID-19 vaccination as a condition of employment for healthcare personnel. Infect Control Hosp Epidemiol 2021. doi: 10.1017/ice.2021.322.

2. Schaffzin J, Shenoy E. Viewpoint: COVID-19 vaccines should be a condition of employment for healthcare personnel. Becker's Hospital Review website. https://www.beckershospitalreview.com/workforce/viewpoint-covid-19vaccines-should-be-a-condition-of-employment-for-healthcare-personnel. html. Published July 14, 2021. Accessed August 6, 2021.

3. Joint statement in support of COVID-19 vaccine mandates for all workers in health and long-term care. American College of Physicians (ACP) website. https://www.acponline.org/acp_policy/statements/ joint_statement_covid_vaccine_mandate_2021.pdf. Published July 26, 2021. Accessed August 8, 2021.

4. VHA directive 1193(1): coronavirus disease 2019 vaccination program for Title 38 healthcare personnel. Department of Veterans' Affairs website. https://www.va.gov/vhapublications/ViewPublication.asp?pub_ID=9355. Amended August 5, 2021. Accessed August 8, 2021.

5. Hanrahan JA, Kammeyer JA, Sievert D, Naylor B, Khuder S, Kaminski B. Implementation of Society for Hospital Epidemiology of America (SHEA) and Centers for Disease Control and Prevention (CDC) Outbreak Response Training Program tools to develop a dedicated coronavirus disease 2019 (COVID-19) hospital in Lucas County, Ohio. Infect Control Hosp Epidemiol 2021. doi: 10.1017/ice.2021.158.

\title{
Severe acute respiratory coronavirus virus 2 (SARS-CoV-2) delta variant of concern breakthrough infections: Are vaccines failing us?
}

\author{
Ali Nowroozi MD² (1) and Nima Rezaei MD, $\mathrm{PhD}^{2,3,4}$ (1) \\ ${ }^{1}$ School of Medicine, Tehran University of Medical Sciences, Tehran, Iran, ${ }^{2}$ Network of Immunity in Infection, Malignancy and Autoimmunity (NIIMA), Universal \\ Scientific Education and Research Network (USERN), Tehran, Iran, ${ }^{3}$ Research Center for Immunodeficiencies, Children's Medical Center, Tehran University of \\ Medical Sciences, Tehran, Iran and ${ }^{4}$ Department of Immunology, School of Medicine, Tehran University of Medical Sciences, Tehran, Iran
}

To the Editor-The severe acute respiratory coronavirus virus 2 (SARS-CoV-2) delta (formerly B.1.617.2) variant of concern (VOC) has become or is becoming the most prevalent type of SARS-CoV-2 in many countries. ${ }^{1}$ Whether available vaccines can protect people against this variant as effectively as against other common variants is yet unknown.

Most countries have already started public vaccination against coronavirus disease 2019 (COVID-19), and the share of people who have received at least 1 vaccine dose reaches as high as $70 \%$ in some regions. ${ }^{2}$ The effect of vaccination is evident in the pattern of daily new cases and deaths in countries with high vaccination rates. ${ }^{3}$ However, many of these nations are experiencing a surge in COVID-19 cases $^{3}$ possibly attributable to novel SARSCoV-2 variants, especially the delta VOC. On the other hand, number of deaths has not increased significantly, ${ }^{3}$ which might be due to the effect of vaccines against serious illness or the relatively short time since the dominance of the new variant. Whether currently available vaccines stand the test of time and limit the COVID-19 burden on communities remains to be seen.

Although several studies have indicated reduced neutralizing antibody activity (ie, vaccine effectiveness) against the delta VOC compared with the B.1.17 lineage and the wild-type virus, ${ }^{4}$ only 2 Medline-indexed studies ${ }^{5,6}$ have assessed vaccine efficacy against this variant. Using data from registries in Scotland, Sheikh et $\mathrm{al}^{5}$ estimated vaccine efficacy against symptomatic delta VOC infection 14 days after the second dose for BNT162b2 (Pfizer-BioNTech) and ChAdOx1 (Oxford-AstraZeneca) at 83\% and $61 \%$, respectively. Efficacy regardless of symptom presence was $79 \%$ for BNT162b2 and $60 \%$ for ChAdOx1. Another study

\footnotetext{
Author for correspondence: Prof. Nima Rezaei, E-mail: rezaei_nima@yahoo.com Cite this article: Nowroozi A and Rezaei N. (2022). Severe acute respiratory coronavirus virus 2 (SARS-CoV-2) delta variant of concern breakthrough infections: Are vaccines failing us?. Infection Control \& Hospital Epidemiology, 43: 1754-1755, https://doi.org/10.1017/ice.2021.363
}

in the United Kingdom ${ }^{6}$ indicated $67.0 \%$ efficacy for ChAdOx 1 and $88.0 \%$ for BNT162b2 vaccines against symptomatic delta VOC infection, 2 weeks after the second injection. Vaccine efficacy 21 days after the first dose was low for both ChAdOxl and BNT162b2 (30.0\% and 35.6\%, respectively).

Although these are the only published studies, promising results have also been found in unpublished investigations. A preprint study by Nasreen et $\mathrm{al}^{7}$ conducted in Canada, showed $56 \%, 72 \%$, and $67 \%$ efficacy against symptomatic delta VOC infection for BNT162b2, mRNA-1273 (Moderna), and ChAdOx1, respectively, 2 weeks after the first dose, and $87 \%$ efficacy 7 days after the second dose of BNT162b2. The vaccines demonstrated $78 \%-96 \%$ protection against serious illness, 14 days after their first dose, which will most likely be even higher after full vaccination.

Most healthcare personnel in Iran have been vaccinated with either ChAdOx1, Gam-COVID-Vac (Sputnik V, Gamaleya), BBIBP-CorV (Sinopharm), or BBV152 (Bharat) vaccine. However, as the country experiences the fifth wave of COVID- $19,{ }^{3}$ there are many reports of infection, serious respiratory involvement, hospitalization, and even death among vaccinated persons. ${ }^{8}$ As indicated in phase 3 trials, ${ }^{9}$ none of the currently available vaccines have $100 \%$ protection against infection, and community protection against serious illness is expected to differ from those of clinical trials. The incidence and severity of breakthrough infections cases is concerning and raises doubts regarding the efficacy of the vaccines utilized against the delta VOC. The proper way of addressing this issue is by conducting studies to assess the efficacy of vaccines against this variant and other VOCs. Unfortunately, to the best of our knowledge, no published or preprint studies have investigated the efficacy of the 4 mentioned vaccines against the delta VOC, except for ChAdOx1. Nonetheless, vaccine producers claim that their vaccines are effective to some degree against the mentioned variant (eg, 90\% for Gam-COVID-Vac and $65.2 \%$ for BBV152). ${ }^{10,11}$

We encourage the scientific community to further evaluate the vaccines that have limited data on their effectiveness (eg, 